\title{
Heights, algebraic dynamics and Berkovich analytic spaces
}

\author{
Jorge Pineiro ${ }^{1}$ \\ Department of Mathematics and Computer Science \\ Bronx Community College of CUNY \\ University Ave. and West 181 Street \\ Bronx, NY 10453 \\ E-mail address: jorge.pineiro@bcc.cuny.edu
}

\begin{abstract}
The present paper is an exposition on heights and their importance in the modern study of algebraic dynamics. We will explain the idea of canonical height and its surprising relation to algebraic dynamics, invariant measures, arithmetic intersection theory, equidistribution and $p$-adic analytic geometry.

AMS Classification 2000: Primary: 14G40; Secondary: 11G50, 28C10, $14 \mathrm{C} 17$.

Keywords: height function, algebraic dynamics, analytic spaces.
\end{abstract}

\section{Introduction}

In many situations, solving mathematical problems involves study the solution set of a system of polynomial equations. The language of algebraic varieties is developed for that purpose. An algebraic variety is a topological locally ringed space whose underlying topological space "behave locally like" the zero set of a system of polynomials in an affine space. A formal definition can be found for example in Chapter 1 of [18]. A projective variety is an algebraic variety that can be embedded into some projective space. Many number theoretic questions are naturally expressed as diophantine problems. Suppose that the algebraic variety $X$ is defined over the number field $F$. An algebraic dynamical system $\varphi: X \rightarrow X$ is a finite map from the algebraic variety $X$ to itself. One of the tools to study algebraic dynamics over number fields is the definition of height functions. A

\footnotetext{
${ }^{1}$ I am very thankful to the organizers of the "XVII Coloquio latinoamericano de Algebra" for the opportunity to participate and present this work.

The author was partially supported by PSC-CUNY Award 69333-00 38.
} 
(canonical) height function associated to $\varphi$ is a function $\hat{h}_{\varphi}: X(\bar{F}) \rightarrow \mathbb{R}$, that attempts to compute the complexity of the point $P \in X(\bar{F})$ relative to the map $\varphi$. For instance the points of finite forward orbits for $\varphi$ will be exactly the points of height $\hat{h}_{\varphi}(P)=0$. The notion of height can be also generalized to subvarieties $Y \subset X$, still measuring the behavior under iteration. The existence of a map from an algebraic variety to a projective space and the construction of the height associated to a self map, both relate to the concept of line bundle. A line bundle is a variety $\mathcal{L}$ together with a map $\pi: \mathcal{L} \rightarrow X$, in such a way that for some open covering $\left\{U_{i}\right\}$ of $X$, the restriction $\mathcal{L} \mid U_{i}=\pi^{-1}\left(U_{i}\right)$ is isomorphic to $U_{i} \times \mathbb{A}_{F}^{1}$. The existence of a morphism from $X$ to a projective space can be expressed in terms of line bundles. A line bundle $\mathcal{L}$ generated by global sections $s_{0}, \ldots, s_{r}$, determines a map to the some projective space $\mathbb{P}^{r}$. Sufficient conditions for the construction of a height relative to a dynamical system can also be expressed in terms of the existence of certain line bundles on $X$. A line bundle $\mathcal{L}$ that satisfies an equation of the sort $\varphi^{*} \mathcal{L} \cong \mathcal{L}^{\otimes \alpha}$ for $\alpha>1$, allows to build a height $\hat{h}_{\varphi}$ relative to the self map $\varphi: X \rightarrow X$. The existence of a line bundle as before is called a polarization for the dynamical system $\varphi$. A polarized dynamical system has associated a canonical function $\hat{h}_{\varphi}$ and a canonical invariant measure $d \mu_{\varphi, v}$ on the analytic space $X_{v}^{a n}$, for each place $v$ of $F$. For places at infinity the canonical measure will be the product of positive currents on $X_{v}^{a n}=X_{\sigma}=X \times_{\sigma} \mathbb{C}$. For finite places, the canonical measure will be defined over the Berkovich analytic space $X_{v}^{a n}=X_{B e r}^{v}$ [5] corresponding to the place in question. A Mahler formula for the height $\hat{h}_{\varphi}$ will compute the height using integration against the canonical measure. The general Mahler formula for a map $\varphi: X \rightarrow X$ is a consequence of the work of Chambert-Loir and Thuillier in theorem 1.3 of [11]. The Mahler formula over curves was first considered in [24] without the tools of Berkovich spaces. On the Riemann sphere the formula reads:

Theorem 1.1. Suppose that $\varphi: \mathbb{P}^{1} \rightarrow \mathbb{P}^{1}$ is a map on $\mathbb{P}_{F}^{1}$ and $f(T) \in$ $\mathcal{O}_{F}(T)$ is a polynomial equation. The height $\hat{h}_{\varphi}(\operatorname{Div}(f))$ can be expressed as sum of integrals over all places $v$ of $F$ of the log of $f(x)$ against the invariant measure $d \mu_{\varphi, v}$, that is,

$$
\hat{h}_{\varphi}(\operatorname{Div}(f))=\sum_{v} \int_{\mathbb{P}_{v, a n}^{1}} \log |f(x)| d \mu_{\varphi, v} .
$$

An important property of the measures $d \mu_{\varphi, v}$ is the equidistribution property. Let's fix a place $v$ of $F$ and an embedding $F_{v} \hookrightarrow \mathbb{C}_{p}$. A sequence $\left\{P_{n}\right\}$ of points in $X(\bar{F})$ is generic if for any subvariety $V \subset X$ there exist $N$ such that $P_{n} \notin V$ for $n>N$. Let $(X, \mathcal{L}, \varphi, \alpha)$ be a polarized dynamical system 
defined over the number field $F$. A sequence of points $\left\{P_{n}\right\} \in X(\bar{F})$ is said to be small if $h_{\varphi}\left(P_{n}\right)$ converges to zero.

Theorem 1.2. (Yuan [29]) Let $(X, \mathcal{L}, \varphi, \alpha)$ be a polarized dynamical system on the projective variety $X$ defined over the number field $F$. Let $\left\{P_{n}\right\}$ a sequence of points on $X(\bar{F})$ which is generic and small, then for any place $v$ of $F$ the Galois orbits of the sequence $\left\{P_{n}\right\}$ are equidistributed in the analytic space $X_{v}^{a n}$ with respect to the invariant measure $\mu_{\varphi, v}$, in the sense that $\frac{1}{\#\left(O\left(P_{n}\right)\right)} \sum_{P \in O\left(P_{n}\right)} \delta_{P}$ converges weakly to $d \mu_{\varphi, v}$ on $X_{v}^{a n}$.

1.1. Notation. Throughout this paper, $X$ will denote a projective algebraic variety of dimension $n$ and $\varphi: X \rightarrow X$ a finite self-map. $F$ will denote a number field and $K$ an algebraically closed field which is complete with respect to a non-archimedean absolute value |.|. $\mathcal{L}$ will denote a line bundle on the algebraic variety $X$. A metrized line bundle $(\mathcal{L},\|\cdot\|)$ on $X$ will be denoted by $\overline{\mathcal{L}}$. The variety $X_{v}$ will denote the variety obtained from $X$ by extension of $F$ to the algebraically closed and complete field $\mathbb{C}_{p}$. The Berkovich analytic space attached to $X_{v}$ for a place $v$ of $F$ will be denoted by $X_{B e r}^{v}$.

\section{Canonical heights and canonical measures}

Height functions are a tool to study dynamics over number fields. As our first approach to height functions we take a look at the naive height on the projective space. The naive height computes the complexity of the point $P \in \mathbb{P}_{\overline{\mathbb{Q}}}$. Suppose that $P \in \mathbb{P}_{F}^{n}$. The naive height represents a variation of the function $\log \max \left(\left|x_{0}\right|, \ldots,\left|x_{n}\right|\right)$, weighted over all absolute values on $F$.

Definition 2.1. The naive height $h_{n v}(P)$ of a point $P=\left(x_{0}, \ldots, x_{n}\right) \in \mathbb{P}_{\overline{\mathbb{Q}}}^{n}$ is given by

$$
h_{n v}\left(x_{0}, \ldots, x_{n}\right)=\frac{1}{[F: \mathbb{Q}]} \sum_{v} \log \max \left(\left|x_{0}\right|_{v}, \ldots,\left|x_{n}\right|_{v}\right)^{N_{v}},
$$

where $v$ is running over all places of $F, F_{v}$ denotes the completion at $v$ and $N_{v}=\left[F_{v}: Q_{p}\right]$ for $v / p$.

Let $X$ be a non-singular algebraic variety defined over $F$ and $\mathcal{L}$ a line bundle on $X$. Assume that the global sections $s_{0}, \ldots, s_{r} \in \Gamma(X, \mathcal{L})$ define a map $\phi_{\mathcal{L}}=\left(s_{0}, \ldots, s_{r}\right): X \rightarrow \mathbb{P}^{r}$. A height $h_{\mathcal{L}}$ associated to $\mathcal{L}$ could be defined by $h_{X, \mathcal{L}}(P)=h_{n v}\left(\phi_{\mathcal{L}}(P)\right)$ for all points $P \in X(\bar{F})$. This definition however depend on the selection of the sections. We can construct a height function $h_{\mathcal{L}}$ associated to every element of $\mathcal{L} \in \operatorname{Pic}(X) \otimes \mathbb{R}$. It will be unique up a bounded function $O(1)$ on $X$ and it will satisfy: 
(i) $h_{\mathcal{L}}$ is $\mathbb{R}$-linear.

(ii) If $X=\mathbb{P}^{n}$ and $\mathcal{L}=\mathcal{O}_{\mathbb{P}^{n}}(1)$, then $h_{\mathcal{L}}=h_{n v}+O(1)$.

(iii) If $f: X \rightarrow Y$ is a morphism of non-singular algebraic varieties and $\mathcal{L} \in \operatorname{Pic}(Y) \otimes \mathbb{R}$, then $h_{X, f^{*} \mathcal{L}}=h_{Y, \mathcal{L}} \circ f+O(1)$.

Definition 2.2. Let $\varphi: X \rightarrow X$ a self-map of $X$ defined over $F$ and let $\mathcal{L} \in \operatorname{Pic}(X) \otimes \mathbb{R}$ be a line bundle on $X$ such that for some number $\alpha>1$ we have $\varphi^{*} \mathcal{L} \cong \mathcal{L}^{\otimes \alpha}$. Such a dynamical system is called a polarized $d y$ namical system $(X, \varphi, \mathcal{L}, \alpha)$. The canonical height associated to a polarized dynamical system is given by

$$
\hat{h}_{\varphi}(P)=\lim _{n \rightarrow \infty} \frac{h_{\mathcal{L}}\left(\varphi^{n}(P)\right)}{\alpha^{n}} .
$$

The condition $\alpha>1$ ensures the existence of the limit. The proof can be found for example in [19], theorem B.4.1. The canonical height satisfy the following properties:

(i) $h_{\varphi}(\varphi(P))=\alpha h_{\varphi}(P) \quad \forall P \in X(\bar{F})$

(ii) $\left|h_{\varphi}(P)-h_{\mathcal{L}}(P)\right|$ is bounded on $X(\bar{F})$.

If $\mathcal{L}$ is ample we can also have the arithmetic properties:

(iii) $h_{\varphi}$ satisfies Northcott's theorem: points with coordinates in $\bar{F}$ with bounded degree and bounded height are finite in number.

(iv) $h_{\varphi}$ is a non-negative function.

(v) $h_{\varphi}(P)=0$ if and only if $P$ has a finite forward orbit by the map $\varphi$. Points with this property are called preperiodic points.

(vi) Lehmer Question: Is there a constant $c_{\varphi}>0$ such that if $P$ is not preperiodic, then $h_{\varphi}(P)>c_{\varphi} / \operatorname{deg}(P)$ ?

In a similar way as we did with the height, we can introduce the canonical invariant measure. Invariant measures were first studied by Brolin [8] and Lyubich [21] for maps on the Riemann sphere and later extended by Brien and Duval [7] to projective spaces of higher dimension. Suppose that $(X, \varphi, \mathcal{L}, \alpha)$ is a polarized dynamical system defined over a number field $F$. Suppose that $\sigma: F \hookrightarrow \mathbb{C}$ is a place of $F$ over infinity. Let $d \mu_{0}$ be a smooth probability measure on $X_{\sigma}=X \times_{\sigma} \mathbb{C}$ and consider the sequence of probability measures on $X_{\sigma}$ recursively defined by $d \mu_{k}=\frac{\varphi^{*} d \mu_{k-1}}{\alpha^{\operatorname{dim}(X)}}$.

Proposition 2.3. The sequence $\left\{d \mu_{k}\right\}$ converge as long as $X_{\sigma}$ is a smooth variety. The limit $d \mu_{\varphi, \sigma}=\lim _{k} d \mu_{k}$ is called the canonical invariant measure on $X_{\sigma}$ relative to $(X, \varphi, \mathcal{L}, \alpha)$. 
The proof of the proposition is outlined in th.3.2.1 of [33]. The work over $\mathbb{P}^{n}$ is done in [7] and some work in the general case is done in [15]. The canonical measure satisfy the following properties:

(i) $\varphi^{*} d \mu_{\varphi, \sigma}=\alpha^{\operatorname{dim}(X)} d \mu_{\varphi, \sigma}$ (functional equation).

(ii) $\varphi_{*} d \mu_{\varphi, \sigma}=d \mu_{\varphi, \sigma}$ (invariance).

(iii) The measure $d \mu_{\varphi, \sigma}$ is a probability measure on $X$.

(iv) The line bundle $\mathcal{L}_{\sigma}=\mathcal{L} \otimes_{\sigma} \mathbb{C}$ can be equipped (th 2.2 in [31]) with a canonical semi-positive metric $\|\cdot\|_{\varphi, \sigma}$. The canonical invariant measure is the product [12] [13] [14] of positive curvature currents $c_{1}(\overline{\mathcal{L}})^{n}=\frac{1}{(\pi i)} \partial \bar{\partial} \log \|\cdot\|_{\varphi, \sigma} \cdots \frac{1}{(\pi i)} \partial \bar{\partial} \log \|\cdot\|_{\varphi, \sigma}$.

\section{Examples and global results for polarized maps}

3.1. Examples. The main source of examples of polarized dynamical systems is given by maps on projective spaces and projection of maps on Tori.

Example 3.1. Power maps on $\mathbb{P}^{n}$.

Consider any of the maps $\phi_{m}: \mathbb{P}_{F}^{n} \rightarrow \mathbb{P}_{F}^{n}$, given by $\phi_{m}\left(x_{0}, \ldots, x_{n}\right)=$ $\left(x_{0}^{m}, \ldots, x_{n}^{m}\right)$ with $m>1$ and the line bundle $\mathcal{O}(1)$ in $\mathbb{P}_{F}^{n}$. The ample line bundle $\mathcal{O}(1)$ in $\mathbb{P}_{F}^{n}$ satisfies $\phi_{m}^{*} \mathcal{O}(1) \cong \mathcal{O}(m)$. The canonical height associated to $\phi_{m}$ is the naive height $h_{n v}$ and the invariant measure is the Haar measure $d \mu$ on the Torus $S^{1} \times \ldots \times S^{1}$. If $T_{0}, T_{1}, \ldots, T_{n}$ represent projective coordinates in $\mathbb{P}^{n}$, the canonical metric at infinity whose curvature gives the canonical current is

$$
\left\|\left(\lambda_{0} T_{0}+\ldots+\lambda_{n} T_{n}\right)\left(a_{0}: \ldots: a_{n}\right)\right\|_{n v}=\frac{\left|\lambda_{0} a_{0}+\ldots+\lambda_{n} a_{n}\right|}{\sup \left(\left|a_{0}\right|, \ldots,\left|a_{n}\right|\right)} .
$$

We can verify the identities:

$$
h_{n v}\left(\phi_{m}(P)\right)=m h_{n v}(P) \quad h_{n v}(P) \geq 0 \quad \phi_{m}^{*} d \mu=d \mu .
$$

Example 3.2. Multiplication by $n$ on elliptic curves.

An elliptic curve is a curve that is at the same time a group object in the category of algebraic varieties. When working over the complex numbers an elliptic curve can be identified with the quotient $\mathbb{C} / \mathbb{Z}+\tau \mathbb{Z}$ for some $\tau$ in the upper half-plane $\mathbb{H}$. As a group it admits self maps representing the multiplication by the different integers. Suppose that we denote the multiplication by $n$ map by $[n]: E \rightarrow E$. Any ample symmetric line bundle $\mathcal{L}$ on $E$ satisfies the equation $[n]^{*} \mathcal{L} \cong \mathcal{L}^{n^{2}}$. The associated canonical height $h_{[n]}$ can be defined for $n>1$ and is the so-called Néron-Tate height $h_{N T}$ on $E$. The canonical measure is given by the normalized Haar measure on the group. 
Example 3.3. Automorphisms on a $K 3$ surface.

This example is due to Silverman [25]. Consider the family $S_{a, b} \subset \mathbb{P}_{F}^{2} \times \mathbb{P}_{F}^{2}$ of algebraic surfaces defined by $\left\{\left(\left(x_{0}, x_{1}, x_{2}\right),\left(y_{0}, y_{1}, y_{2}\right)\right): \sum_{i, j=0}^{2} a_{i, j} x_{i} y_{j}=\right.$ $\left.\sum_{i, j, k, l=0}^{2} b_{i, j, k, l} x_{i} x_{k} y_{j} y_{l}=0\right\}$. The natural projections $p_{1}, p_{2}: S_{a, b} \rightarrow \mathbb{P}^{2}$, represents $2: 1$ coverings of $\mathbb{P}^{2}$ and determine involutions $\sigma_{1}$ and $\sigma_{2}$. The group generated by these involutions is an infinite group of automorphisms on generic members of the family $S_{a, b}$. Denote by $L_{i}=p_{i}^{*} \mathcal{O}_{\mathbb{P}^{2}}(1), E^{+}=$ $(2+\sqrt{3}) L_{1}-L_{2}$ and $E^{-}=-L_{1}+(2+\sqrt{3}) L_{2}$, the geometry of the family $S_{a, b}$ can be used to prove that $\left(\sigma_{1} \circ \sigma_{2}\right)^{ \pm 1 *}\left(E^{ \pm}\right)=(7+4 \sqrt{3}) E^{ \pm}$and we obtain two canonical heights $\hat{h}^{ \pm}$associated to $E^{ \pm}$and $\left(\sigma_{1} \circ \sigma_{2}\right)^{ \pm 1}$. The polarizing line bundles $E^{ \pm}$are not ample. One can get $\left(E^{ \pm}, E^{ \pm}\right)=0$. Another approach to this example can be found in the work of Kawagushi [20].

3.2. Polarized dynamical systems on smooth projective varieties. Denote by $\Omega_{X}$ the canonical line bundle on the smooth variety $X$ and by $K_{X}$ its associated canonical divisor. Assuming that the linear system $\left|m K_{X}\right|$ is not empty we can look at the associated rational map $\phi_{\Omega_{X}^{m}}$ : $X \rightarrow \mathbb{P}^{l}$. We define $g_{m}=\operatorname{dim}\left(\phi_{\Omega_{X}^{m}}(X)\right)$ and the Kodaira dimension $\kappa(X)$ as $\kappa(X)=\sup _{m \geq 1} g_{m}$. If all $g_{m}=0$, the Kodaira dimension $\kappa(X)$ is defined as $\kappa(X)=-1$. The Kodaira dimension becomes an obstruction to the existence of polarized dynamical systems on an algebraic variety.

Lemma 3.4. Let $(X, \varphi, \mathcal{L}, \alpha)$ be a polarized system defined over $F$. Assume that the line bundle $\mathcal{L}$ is ample and $X$ is smooth, then $\alpha^{\operatorname{dim}(X)}=\operatorname{deg}(\varphi)$.

Proof. Let $D$ be a divisor such that $\varphi^{*} D \equiv \alpha D$, let $D^{k}$ denotes the selfintersection of $D$ with itself $\mathrm{k}$ times and finally let's put $\operatorname{dim}(X)=n$. We have

$$
\alpha^{n}\left(D^{n-1}, D\right)=\left(\left(\varphi^{*} D\right)^{n-1}, \varphi^{*} D\right)=\varphi^{*}\left(D^{n-1}, D\right)=\operatorname{deg}(\varphi)\left(D^{n-1}, D\right) .
$$

The self-intersection $\left(D^{n-1}, D\right) \neq 0$ because $\mathcal{L}_{D}$ is ample.

Theorem 3.5. If $(X, \varphi, \mathcal{L}, \alpha)$ is a polarized dynamical system with $\mathcal{L}$ ample and $X$ smooth, then $\kappa(X) \leq 0$.

Proof. The proof is taken from [33], proposition 2.1.1 part 1. Let's denote by $R_{\varphi}$ the ramification divisor of $\varphi: X \rightarrow X$. Then we have $R_{\varphi}=K_{X}-$ 
$\varphi^{*} K_{X}$ because the map is separable and

$$
\begin{aligned}
\mathcal{L}^{n-1} R_{\varphi} & =\mathcal{L}^{n-1} K_{X}-\mathcal{L}^{n-1} \varphi^{*} K_{X} \\
& =\mathcal{L}^{n-1} K_{X}-\alpha^{1-n} \varphi^{*}\left(\mathcal{L}^{n-1} K_{X}\right) \\
& =\mathcal{L}^{n-1} K_{X}-\alpha \mathcal{L}^{n-1} K_{X}=(1-\alpha)\left(\mathcal{L}^{n-1} K_{X}\right)
\end{aligned}
$$

The first step in the equations is using the polarization property and the second step is using lemma 3.4. Now, if $\kappa(X)>0$, then $m K_{X} \neq 0$ is effective for some $m$, and therefore $\left(\mathcal{L}^{n-1} K_{X}\right)>0$. That contradicts the facts $\mathcal{L}^{n-1} R_{\varphi} \geq 0$ and $\alpha>1$.

Remark 3.6. Polarized maps on Surfaces (proposition 2.3.1 of [33]).

Assume that $X$ is a smooth variety of dimension two. Suppose that the hypothesis of the previous theorem are valid. If $\kappa=0$ and $\alpha$ is an integer, a theorem of Beauville ([4], theorem 1$)$ will characterize the universal covering $\bar{X}$ of $X$. As a result $X \cong T / G$ where $T$ is a complex torus and $G$ is a finite group. In this case the map $\varphi$ will be induced by a linear map $\bar{\varphi}: \mathbb{C}^{n} \rightarrow \mathbb{C}^{n}$. If $\kappa<0$ we have to consider rational surfaces and irrational ruled surfaces. A theorem of Nakayama ([22], theorem 3) will prove that a rational surface has an endomorphism of degree $\geq 2$ if and only if it is toric. The other possibility are $\mathbb{P}^{1}$-bundles $\pi: X \rightarrow C$ where $C$ has genus greater that zero. In fact we can reduce to consider $C=E$ an elliptic curve.

3.3. Mahler formula. For a general map $\varphi: \mathbb{P}^{1} \rightarrow \mathbb{P}^{1}$, we can always consider a polarization by the line bundle $\mathcal{O}(1)$, as long as $\alpha=\operatorname{deg}(\varphi)>1$. The following result relates the canonical height and the canonical measure.

Theorem 3.7. If $\varphi: \mathbb{P}^{1} \rightarrow \mathbb{P}^{1}$ is given in projective coordinates by a monic polynomial, we can express the height $\hat{h}_{\varphi}(P)$ of a point $P \in \mathbb{P}_{\overline{\mathbb{Q}}}^{1}$ as sum of integrals over all places $\sigma$ at infinity of the log of the minimal polynomial for $P$ against the invariant measure $d \mu_{\varphi, \sigma}$.

$$
\hat{h}_{\varphi}(P)=\frac{1}{\operatorname{deg}(P)} \sum_{\sigma} \int_{X(\mathbb{C})} \log |f(x)| d \mu_{\varphi, \sigma}
$$

The proof can be found in [24] or [23]. It uses the theory of adelic metrized line bundles developed by S.Zhang in [31] and the Arakelov intersection theory explained in [27]. Metrized line bundles will be treated with more details later in this paper. A metric $\|$.$\| on a line bundle \mathcal{L}$ is a norm on each of the fibres, varying continuously. Adelic metrized line bundles are provided with a collection of metrics $\|.\|_{v}$, where $v$ runs over all places of a number field $F$. The height of a cycle can be defined relative to any 
adelic metric. In particular we are interested in the height relative to the canonical adelic metric $\|.\|_{\varphi}$ of th. 2.2 in [31].

$$
\hat{h}_{\varphi}(Y)=\frac{\operatorname{deg}\left(c_{1}\left(\mathcal{L},\|\cdot\|_{\varphi}\right)^{\operatorname{dim}(Y)+1} \mid Y\right)}{(\operatorname{dim}(Y)+1)\left(c_{1}(\mathcal{L})^{\operatorname{dim}(Y)}\right.} .
$$

In this way when $Y=P$ we recover the canonical height of a point via adelic intersection. Using the symmetry and the recursive definition of dêg, the canonical height of a principal cycle $\operatorname{Div}(f)$ can be expressed by the formula $\hat{h}_{\varphi}(\operatorname{Div}(f))=\sum_{\sigma} \int_{X(\mathbb{C})} \log |f(x)| d \mu_{\varphi, \sigma}$ and the result follows.

Suppose that we impose no condition on the map $\varphi=(P, Q): \mathbb{P}_{F}^{1} \rightarrow \mathbb{P}_{F}^{1}$, it may happen that the polynomials $P$ an $Q$ have common roots and therefore the map can not be extended to the integral model $\mathbb{P}_{\mathcal{O}_{F}}^{1}$. The formula will need an error term $E$ ( $f$,vfinite) depending on finite places of bad reduction, that is, the set of places $v$ where the map does not extend to a well defined map on $\mathbb{P}_{v}^{1}$. We get something like,

$$
\hat{h}_{\varphi}(\operatorname{Div}(f))=\sum_{\sigma} \int_{\mathbb{P}^{1}(\mathbb{C})} \log |f(x)| d \mu_{\varphi, \sigma}+E(f, \text { vfinite }) .
$$

Intersection theory can give us an idea of the nature of the term $E(f, v$ finite $)$. Suppose that we denote by $Y$ the closed subscheme of $X=\mathbb{P}_{\mathcal{O}_{F}}^{1}$ determined by the vanishing of $P$ and $Q$. Suppose also that $I$ is the sheaf of ideals defined by $Y$. Then we have a surjection $\varphi_{1}: \mathcal{O}_{X}^{2} \rightarrow I(d)$, where $d=\operatorname{deg}(P)=\operatorname{deg}(Q)$. The scheme-theoretic image of the projection from $Y$ to $\operatorname{Spec}\left(\mathcal{O}_{F}\right)$ is called "places of bad reduction". If we denote by $\sigma: X_{1} \rightarrow X$ the blow-up of $Y$ in $X$, we will obtain that for a positive Cartier divisor $E_{1}$, the pullback $\sigma^{*} I=\mathcal{O}_{X_{1}}\left(-E_{1}\right)$, this give rise to a surjective map $\mathcal{O}_{X_{1}}^{2} \rightarrow \sigma^{*}\left(\mathcal{O}_{X}(d)\right) \otimes \mathcal{O}_{X_{1}}\left(-E_{1}\right)$. By the universal property of the projective line this gives a map $\varphi: X_{1} \rightarrow X$, extending the original $\varphi: X \rightarrow X$. We can repeat this process with $\varphi^{k}$ in place of $\varphi$ and obtain a model $\sigma_{k}: X_{k} \rightarrow X$ and a map $\varphi_{k}: X_{k} \rightarrow X$. The Cartier divisor $E_{k}$ is the sum of connected components $E_{k}=\sum_{v \in N, j} r_{v, j, k} C_{v, j, k}$. The error term $E(f, v$ finite $)$ in the Mahler formula can be obtained as limit of the intersection numbers $\left(\varphi_{k}^{*} \mathcal{O}(1), \sigma_{k}^{*} \operatorname{Div}(f)\right)_{X_{k}}$.

The methods described above could be extended to study morphisms on the $n$-dimensional projective space [23]. It is very interesting however to see a completely different approach to compute $E(f, v$ finite $)$ using the concept of $p$-adic analytic spaces. Suppose that $v$ is a finite place of $F$. It will be nice to embed the reduction $X\left(F_{v}\right)$ in the appropriated analytic space $X_{v}^{a n}$ and define a measure $d \mu_{\varphi, v}$ on it. The idea would be to recover the error term $E$ (f, vfinite $)$ as sum of integrals over finite places. In this way we 
could express our formula in the more symmetric way

$$
\hat{h}_{\varphi}(\operatorname{Div}(f))=\sum_{v} \int_{X_{v}^{a n}} \log |f(x)| d \mu_{\varphi, v} .
$$

This work is carried out in [11]. Suppose that $(X, \varphi, \mathcal{L}, \alpha)$ is a polarized dynamical system on the $\mathrm{n}$-dimensional projective variety $X$. Suppose that the section $s_{D}$ corresponds to the divisor $D$ in $X$ and $f_{D}=s_{D} / x_{0}^{\operatorname{deg}(D)}$. We can express the height of $\operatorname{Div}\left(f_{D}\right)$ as sum of integrals

$$
\hat{h}_{\varphi}\left(\operatorname{Div}\left(f_{D}\right)\right)=\sum_{v} \int_{X_{v}^{B e r}} \log \left|f_{D}(x)\right| d \mu_{\varphi, v},
$$

where the $v$ is running over all places of $F$. For finite places the $X_{v}^{B e r}$ is the Berkovich analytic space [5] associated to $v$, and $d \mu_{\varphi, v}$ is the invariant measure on $X_{v}^{B e r}$ introduced in [10]. The theory of Berkovich analytic spaces is a continuation of the theory of rigid analytic spaces initiated by Tate. Tate defined rigid analytic space and associated to any scheme $X$ over a complete field $K$, a space $X^{\text {rig }}$ that allows to define coherent sheafs and cohomology similar to the geometric situation $K=\mathbb{C}$. Berkovich spaces make possible the definition of path-connection and harmonic functions. In the context of $p$-adic analytic spaces, the Berkovich spaces make possible to do measure theory.

\section{Berkovich analytic spaces}

Let $K$ an algebraically closed field which is complete with respect to a non-archimedean absolute value |.|. The topology of $K$ induced by its absolute value is totally disconnected and that makes it difficult to establish notions like continuity, homotopy, Laplace operator and harmonic functions, while working on a scheme $X$ over $K$. In [5] a new special category of locally ringed topological spaces is introduced: The Berkovich $K$-analytic spaces. Berkovich $K$-analytic spaces have some properties similar to analytic manifolds, for example each point in a K-analytic space admits a fundamental system of neighborhoods which are locally compact and path-connected. A very important point is that one can associate with any scheme $X$ of locally finite type over $\mathrm{K}$, a Berkovich $K$-analytic space $X_{B e r}$. The scheme $X$ is separated (resp. proper, resp. connected) if and only if the $X_{B e r}$ is Hausdorff (resp. compact, resp. path-connected). If $X$ is separated its dimension is equal to the topological dimension of $X_{B e r}$. Moreover there is a canonical embedding of $X(K)$ as a dense subspace of $X_{B e r}$. In this way we can embed a connected scheme $X(K)$, with its locally disconnected topology, in the path-connected space $X_{B e r}$, and create a better context 
to define continuity, harmonic functions, etc. The following examples are borrowed from Baker's presentation in [2].

Example 4.1. The Berkovich space associated to an affine scheme.

Consider the affine scheme $X=\operatorname{Spec}(A)$ over $K$. In this case $X$ is associated with the analytic space $X_{B e r}$ whose underlying topological space $\mathcal{M}(A)$ is made out of all multiplicative seminorms on $A$, extending the absolute value on $K$, equipped with the weakest topology for which all functions of the form $|.| \rightarrow|f|$ for $f \in A$ are continuous.

Example 4.2. The Berkovich space $\mathbb{A}_{B e r}^{1}$.

The underlying topological space of $\mathbb{A}_{B e r}^{1}$ is the set $\mathcal{M}(K[T])$ of all multiplicative seminorms on $K[T]$ extending the absolute value on $\mathrm{K}$. For example, whenever we consider a disk $B(a, r)=\{z \in K:|z-a| \leq r\}$ in $\mathrm{K}$, and define $|f|_{B(a, r)}=\sup _{z \in B(a, r)}|f(z)|$, Gauss lemma will imply that $|\cdot|_{B(a, r)}$ is a multiplicative seminorm on $K[T]$. Suppose that $x, y$ corresponds to seminorms $|\cdot|_{B(a, r)}$ and $|\cdot|_{B(b, s)}$ respectively. Lets denote by $x \vee y$ the seminorm associated with the smallest disk $B(a,|b-a|)$ containing both $B(a, r)$ and $B(b, s)$. A path between $x$ and $y$ can be seen as all seminorms associated to disks $B\left(a, r^{\prime}\right)$ where $r \leq r^{\prime} \leq|b-a|$ followed by the seminorms associated to disks $B\left(b, s^{\prime}\right)$ with $|b-a| \geq s^{\prime} \geq s$. Passing from points to seminorms give for the first time a notion of connectivity on $K$. However the general situation is a little bit more complicated because not all points in the affine Berkovich space are associated to disks. The following classification theorem is due to Berkovich.

Theorem 4.3. Every point $x \in \mathbb{A}_{\text {Ber }}^{1}$ corresponds to a nested sequence $B\left(a_{1}, r_{1}\right) \supseteq B\left(a_{2}, r_{2}\right) \supseteq B\left(a_{3}, r_{3}\right) \ldots$, of closed disks in the sense that

$$
|f|_{x}=\lim _{n \rightarrow \infty}|f|_{B\left(a_{n}, r_{n}\right)} .
$$

Two nested sequences define the same point of $\mathbb{A}_{\text {Ber }}^{1}$ if and only if a) each has a nonempty intersection, and their intersections are the same; or

b) both have empty intersection, and the sequence are cofinal.

Example 4.4. Berkovich projective line $\mathbb{P}_{B e r}^{1}$

The Berkovich analytic space $\mathbb{P}_{B e r}^{1}$ is the one-point compactification of $\mathbb{A}_{\text {Ber. }}^{1}$. The Berkovich projective space is homeomorphic to the inverse limit of finite $\mathbb{R}$-trees. If we denote by $\delta_{0,1}$ the point corresponding to the seminorm $|f|_{B(0,1)}=\sup _{z \in B(0,1)}|f(z)|$, there are infinitely many branches of the tree emanating from this point. One branch "moving up" to infinity and the others corresponding to elements in the residue field $\tilde{K}$. Again for any 
point of the branches associated with a disk $|\cdot|_{B(a, r)}$ with rational radius $\left(r \in\left|K^{*}\right|\right)$, infinitely many branches keep emerging. Picture 1 in [2] gives an idea of how the projective Berkovich line looks like.

Example 4.5. Berkovich projective curves of genus at least one.

Let $X$ be a projective curve of genus greater or equal to one. There is canonically defined subset $\Sigma \subset X_{B e r}$, which is homeomorphic to a finite one-dimensional CW-complex, and the entire space $X_{B e r}$ admits a deformation retraction $r$ to $\Sigma$. The fibres $r^{-1}(x)$, for $x \in \Sigma$, are all homeomorphic to topological trees. Picture 4 in [2] provides an example representing the Berkovich analytic space associated to an elliptic curve with multiplicative reduction.

4.1. Metrics on Line bundles. Let $\mathcal{L}$ be a line bundle on $X$. A metric on $\mathcal{L}$ is a continuous function $\|s\|: U \rightarrow \mathbb{R}^{+}$for each open $U \subset X$ and $s \in \Gamma(U, \mathcal{L})$, such that $\|f s\|=|f|\|s\|$ for all continuous function $f: U \rightarrow K$. For example every continuous function on $X$, defines a metric on the trivial line bundle $\mathcal{O}_{X}$, by $\|1\|(x)=\exp (-f(x))$.

Let $X$ be a projective variety over $K$. A model $\tilde{X}$ of $X$ over the ring of integers $K_{0}=\{a \in K:|a| \leq 1\}$ is a proper and flat $K_{0}$-scheme whose generic fibre is $X$. Suppose that we have a projective model $\tilde{X}$ of $X$ over $K_{0}$, and a line bundle $\tilde{\mathcal{L}}$ whose restriction to $X$ is $\mathcal{L}^{m}$ for some $m>0$. We can define a metric on $\mathcal{L}$ as follows: let $x \in X(K)$ and $\tilde{x}: \operatorname{Spec}\left(K_{0}\right) \rightarrow \tilde{X}$ the section extending $x$, then we have $\tilde{x}^{*} \tilde{\mathcal{L}} \otimes_{R} K=x^{*} \mathcal{L}^{m}$ and for each $l \in x^{*}(\mathcal{L})$ we can define

$$
\|l\|_{\tilde{\mathcal{L}}}=\inf _{a \in K}\left\{|a|^{1 / m}: l \in a \tilde{x}^{*} \tilde{\mathcal{L}}\right\} .
$$

A metric on $\mathcal{L}$ is called algebraic if it is defined by a model $\tilde{X}$ of $X$. An algebraic metric is semi-positive if the reduction $\tilde{L}_{s}$ of $\mathcal{L}$ to the special fibre $\tilde{X}_{s}$ has non-negative degree on every curve. By abuse of notation we will call semi-positives, all metrics obtained as uniform limit of semi-positive metrics as before. Let $\mathcal{L}$ be a line bundle over $X$ and denote by $\mathcal{L}^{a n}$ its extension to $X_{B e r}$. Suppose that $(\tilde{X}, \mathcal{L})$ is a model of $(X, L)$ over $K_{0}$. Let's denote by $s p_{\tilde{X}}: X_{B e r} \rightarrow \tilde{X}_{s}$ the reduction map. We can define a metric on the line bundle $L^{a n}$ in the following way: for every open $U$ in $\tilde{X}$ and a trivialization $\varepsilon$ of $\mathcal{L}$ over $\mathrm{U}$, one has $\left\|s p_{\tilde{X}}^{*} \varepsilon\right\|=1$ on $s p_{\tilde{X}}^{-1}\left(U_{s}\right)$. A metrized line bundle $\bar{L}$ on $X$ can be extended in this way to a metrized line bundle $\bar{L}^{a n}$ on the Berkovich space $X_{B e r}$. The same work could be carry out [16] with formal admissible schemes as models $\tilde{X}$. We have the distinguished class of functions: 
Definition 4.6. A continuous function over $X_{\text {Ber }}$ is called a model function if it is equal to $-\log \|1\|^{1 / m}$ for some positive integer $m$ and $\|$.$\| is$ the metric induced by a model $(\tilde{X}, \tilde{\mathcal{L}})$ of $\left(X, \mathcal{L}^{m}\right)$. If we allow $\tilde{X}$ to be an admissible formal scheme [16] we obtain formal metrics and formal model functions.

The following two results allow to restrict the work with continuous functions to the study of formal models and in particular to the completion of global models along the special fibre. These results are applied to prove weak convergence results of measures in $X_{B e r}$.

Theorem 4.7. (Gubler) The vector space of formal model functions on $X_{B e r}$ is uniformly dense in the ring of real continuous valued functions on $X_{B e r}$.

This is theorem 7.12 in [16]. The proof is an application of the StoneWeierstrass theorem on the compact $X_{B e r}$.

Theorem 4.8. All formal metrics over the trivial bundle of $X_{B e r}$ are induced by global projective $K_{0}$-models.

This result is already explained in [10]. A proof can be found in lemma 5.5 in [29]. It uses formal GAGA principle.

4.2. Canonical measures on Berkovich spaces. Let $X$ be a projective variety of dimension $n$. Suppose that we have metrized line bundles $\mathcal{L}_{1}, \ldots \mathcal{L}_{n}$ on $X$, such that the metrics are induced by models $\left(\tilde{X}, \tilde{\mathcal{L}}_{i}\right)$ of $\left(X, \mathcal{L}^{e_{i}}\right)$ for some positive numbers $e_{i}$ over $\operatorname{Spec}\left(K_{0}\right)$. Let's also assume that the model is normal. If $\tilde{X}_{j, s}$ is a connected component of the special fibre $\tilde{X}_{s}$, by proposition 2.4.4 in [5] there exist a unique point $\xi_{j}$ in $X_{B e r}$, such that the reduction $s p_{\tilde{X}}\left(\xi_{j}\right)=$ generic point of $\tilde{X}_{j, s}$. Considering that the component $\tilde{X}_{j, s}$ appears in $\tilde{X}_{s}$ with multiplicity $\eta_{j}$, the semi-positive metrized line bundles $\overline{\mathcal{L}}_{1}, \overline{\mathcal{L}}_{2}, \ldots \overline{\mathcal{L}}_{n}$ on $\tilde{X}$ actually define a measure

$$
c_{1}\left(\overline{\mathcal{L}}_{1}\right) \ldots c_{1}\left(\overline{\mathcal{L}}_{n}\right)=\frac{1}{e_{1} \ldots e_{n}} \sum_{j} \eta_{j}\left(c_{1}\left(\mathcal{L}_{1}\right) \ldots c_{1}\left(\mathcal{L}_{n}\right) \mid \tilde{X}_{j, s}\right) \delta_{\xi_{j}},
$$

on $X_{B e r}$ as linear combination of dirac measures. More generally if $Z \subset X$ is a subvariety of $X$ of dimension $m$, one can define the measure

$$
c_{1}\left(\overline{\mathcal{L}}_{1}\right) \ldots c_{1}\left(\overline{\mathcal{L}}_{m}\right) \delta_{Z}=i_{*} c_{1}\left(\overline{\mathcal{L}}_{1} \mid Z\right) \ldots c_{1}\left(\overline{\mathcal{L}}_{m} \mid Z\right),
$$

where $i: Z_{B e r} \hookrightarrow X_{B e r}$ is the canonical immersion. The regular Borel measure $c_{1}\left(\overline{\mathcal{L}}_{1}\right) \ldots c_{1}\left(\overline{\mathcal{L}}_{n}\right)$ on $X_{B e r}$ satisfy the following properties:

(i) It is multilinear and symmetric in $\left(\mathcal{L}_{1},\|\cdot\|_{1}\right), \ldots,\left(\mathcal{L}_{n},\|\cdot\|_{n}\right)$. 
(ii) If $\varphi: X \rightarrow X^{\prime}$ is a morphism of projective varieties of dimension $n$, then $\varphi_{*} c_{1}\left(\varphi^{*} \overline{\mathcal{L}}_{1}\right) \ldots c_{1}\left(\varphi^{*} \overline{\mathcal{L}}_{n}\right)=\operatorname{deg}(\varphi) c_{1}\left(\overline{\mathcal{L}}_{1}\right) \ldots c_{1}\left(\overline{\mathcal{L}}_{n}\right)$.

(iii) The total mass of the measure is $\operatorname{deg}_{\mathcal{L}_{1}, \ldots, \mathcal{L}_{n}}(X)$.

(iv) Suppose that $\overline{\mathcal{L}}_{0}, \overline{\mathcal{L}}_{1}, \ldots \overline{\mathcal{L}}_{n}$ represent metrized line bundles on $X$. Suppose also that $\overline{\mathcal{L}}_{0}, \overline{\mathcal{L}}_{1}$ are both isomorphic to the trivial line bundle $\mathcal{O}_{X}$ and for $i=0,1$ we denoted $\varphi_{i}=-\log \|1\|_{i}$. Then we obtain the following version of the Stokes formula: $\int_{X_{B e r}} \varphi_{0} c_{1}\left(\overline{\mathcal{L}}_{1}\right) c_{1}\left(\overline{\mathcal{L}}_{2}\right) \ldots c_{1}\left(\overline{\mathcal{L}}_{n}\right)=\int_{X_{B e r}} \varphi_{1} c_{1}\left(\overline{\mathcal{L}}_{0}\right) c_{1}\left(\overline{\mathcal{L}}_{2}\right) \ldots c_{1}\left(\overline{\mathcal{L}}_{n}\right)$.

(v) Suppose that for all i, $\overline{\mathcal{L}}_{i}$ is equipped with a semi-positive metric. For every sequence of algebraic semi-positive metrics $\overline{\mathcal{L}}_{i, k}$ on the $\mathcal{L}_{i}$, converging to the given metric, the sequence of measures $c_{1}\left(\overline{\mathcal{L}}_{1, k}\right) \ldots c_{1}\left(\overline{\mathcal{L}}_{n, k}\right) \delta_{Z}$ converges to a measure on the subvariety $Z$ denoted by $c_{1}\left(\overline{\mathcal{L}}_{1}\right) \ldots c_{1}\left(\overline{\mathcal{L}}_{n}\right) \delta_{Z}$.

Definition 4.9. Let $(\mathcal{L},\|\|$.$) be a semi-positive metrized line bundle on X$. Suppose that the metrized line bundles $\overline{\mathcal{L}}_{1}=\overline{\mathcal{L}}_{2}=. .=\overline{\mathcal{L}}_{n}=\overline{\mathcal{L}}$ are all equal. The probability measure $d \mu_{\overline{\mathcal{L}}}=c_{1}(\overline{\mathcal{L}})^{n} / c_{1}(\mathcal{L})^{n}$ is called the probability measure on $X_{\text {Ber }}$ relative to $\overline{\mathcal{L}}$. Suppose that $(X, \varphi, \mathcal{L}, \alpha)$ is a polarized dynamical system on $X$ and $\mathcal{L}$ is equipped with the canonical metric $\left\|_{0}\right\|_{\varphi}$ introduced in [31], then $d \mu_{\varphi}=d \mu_{\overline{\mathcal{L}}}$ is called the invariant canonical probability measure on $X_{B e r}$.

Remark 4.10. This measures introduced by Chambert-Loir in [10] over discrete valued field were extended in [17] to algebraically closed fields.

Example 4.11. The canonical measure over $\mathbb{P}_{\text {Ber }}^{1}$.

Suppose that $X=\mathbb{P}_{K}^{n}$ and $\mathcal{L}=\mathcal{O}(1)$ is provided with the naive metric. The measure $\mu_{\overline{\mathcal{L}}}$ on $\mathbb{P}_{B e r}^{n}$ is the Dirac measure supported on the canonical point of $\mathbb{P}_{B e r}^{n}$ whose reduction is the generic point of $\mathbb{P}_{K}^{n}$. In the case of $n=1$ it corresponds to the Gauss norm on the formal power series $K\{T\}$.

4.3. Intersection theory. Assume as before that $X$ is an algebraic variety of dimension $n$ over the complete and algebraically closed field $K$. Let $Z \in Z_{d}(X)$ be a cycle of dimension $d$ on $X$. Let $\overline{\mathcal{L}}_{0}, \ldots, \overline{\mathcal{L}}_{d}$ be a set of hermitian line bundles [31] provided with semi-positive metrics $\|\cdot\|_{i}$ on $X$. Assume that the sections $s_{i}$ of $\mathcal{L}_{i}$ intersect properly on $Z$. In the same spirit of the Arakelov intersection theory [27], the arithmetic intersection number $\operatorname{deg}_{Z}\left(\hat{c}_{1}\left(\mathcal{L}_{0}\right) \ldots \hat{c}_{1}\left(\mathcal{L}_{d}\right) \mid Z\right) \in \mathbb{R}$ relates to the measure $c_{1}\left(\overline{\mathcal{L}}_{1}\right) \ldots c_{1}\left(\overline{\mathcal{L}}_{d}\right) \delta_{Z}$ by the recursive equation:

$$
\begin{aligned}
\operatorname{deg}_{Z}\left(\hat{c}_{1}\left(\mathcal{L}_{0}\right) \ldots \hat{c}_{1}\left(\mathcal{L}_{d}\right) \mid Z\right)= & \hat{\operatorname{deg}}_{Z}\left(\hat{c}_{1}\left(\mathcal{L}_{1}\right) \ldots \hat{c}_{1}\left(\mathcal{L}_{d}\right) \mid Z \cdot \operatorname{Div}\left(s_{0}\right)\right) \\
& -\int_{X_{B e r}} \log \left\|s_{0}\right\|_{d} c_{1}\left(\overline{\mathcal{L}}_{1}\right) \ldots c_{1}\left(\overline{\mathcal{L}}_{d}\right) \delta_{Z} .
\end{aligned}
$$


For $k=0$ and $Z=\sum_{i} n_{i} P_{i}\left(P_{i} \in X_{v}\right)$, we have $\hat{\operatorname{deg}}_{Z}=\sum_{i} n_{i} N_{P_{i}} \log N(v)$ where $N_{P_{i}}=\left[K\left(P_{i}\right): K\right]$. A similar expression is obtained when $X$ is a complex analytic variety and we interpret $c_{1}\left(\overline{\mathcal{L}}_{i}\right)$ as the curvature current relative to the metric, and $\delta_{Z}$ as the integration current on $Z$.

4.4. The Blow-up and the Berkovich analytic space. Suppose that $X=\mathbb{P}_{\mathcal{O}_{F}}^{1}$ and $\varphi=(P, Q): \mathbb{P}^{1} \rightarrow \mathbb{P}^{1}$ is a self-map of degree $d>1$. In previous sections we were able to build models $\sigma_{k}: X_{k} \rightarrow X$ as blow-ups of subschemes $Y_{k}$ of $X$. The map $\varphi^{k}: X \rightarrow X$ could then be extended to a map $\varphi_{k}: X_{k} \rightarrow X$ and the exceptional divisor was written as $E_{k}=$ $\sum_{v \in N, j} r_{v, j, k} C_{v, j, k}$. Suppose that the line bundle $\mathcal{O}(1)$ on $X$ is endowed with the naive metric $\|.\|_{0}$ at all places. The line bundles $\mathcal{L}_{k}=\varphi_{k}^{*} \mathcal{O}(1)$ can be endowed with metrics $\|\cdot\|_{v, k}$ at every place with the property that $\|\cdot\|_{v, k}=\varphi_{k}^{*}\|\cdot\|_{v, 0}^{1 / d^{k}}$. The pair $\left(X_{k}, \overline{\mathcal{L}}_{k}\right)$ defines a measure $d \mu_{\overline{\mathcal{L}}_{v, k}}$ on the Berkovich space $X_{v}^{B e r}$. The sequence of metrics $\|\cdot\|_{k}$ converges to a semipositive metric $\|\cdot\|_{v, \varphi}$ in the sense of [31] and as a consequence we have the convergence of measures $d \mu_{\overline{\mathcal{L}}_{v, k}}$ to a measure $d \mu_{v, \varphi}$ on $X_{v}^{B e r}$, and the convergence of the heights $h_{\overline{\mathcal{L}}_{k}}$ to $h_{\varphi}$.

Suppose that we have a polynomial $f(T)$ and $\operatorname{Div}(f)=D-\operatorname{deg}(f) \infty+$ $\sum_{\text {finite } v} v(f) X_{v}$. Let us denote by $f_{k}=\sigma_{k}^{*} f$ and $D_{k}$ the proper transform of $D$ by the map $\sigma_{k}$. Recall that proposition 3.10 in [23] gives $\left(\overline{\mathcal{L}}_{k}, \operatorname{Div}\left(f_{k}\right)\right)=$ $d^{k} \sum_{v \mid \infty} \int_{\mathbb{P}^{1}\left(\mathbb{C}_{v}\right)} \log |f| d \mu_{v, k}$. Also the intersection formula on $\mathbb{P}_{v, \text { Ber }}^{1}$ gives $\left.d^{k} \int_{\mathbb{P}_{v, \text { Ber }}^{1}} \log |f| d \mu_{v, k}=\operatorname{deg}\left(\hat{c}_{1}\left(\overline{\mathcal{L}}_{k}\right)\right) \mid \operatorname{Div}\left(f_{k}\right)\right)$.

\section{Equidistribution theorems}

The application of arithmetic intersection and height theory to proof results of equidistribution was first considered in [26]. The work is done there for Abelian varieties defined over number fields and places over infinity. For the general case consider the algebraic variety $X$ defined over the number field $F$. Let's fix a place $v$ of $F$ and an embedding $F_{v} \hookrightarrow \mathbb{C}_{p}$. Let $\left\{P_{n}\right\}$ be a sequence of points on $X(\bar{F})$ and let $\mu_{v}$ be a probability measure on the associated analytic space $X_{v}^{a n}$. In archimedean case it represents the complex analytic space $X\left(\mathbb{C}_{v}\right)$ and in the ultrametric case the Berkovich analytic space $X_{B e r, v}$.

Definition 5.1. We say that the orbits $\left\{O\left(P_{n}\right)\right\}$ of the sequence $\left\{P_{n}\right\}$ under the action of $\operatorname{Gal}(\bar{F} / F)$ are equidistributed with respect to a measure $\mu_{v}$, when the probability measures $\mu_{v, n}=\frac{1}{\#\left(O\left(P_{n}\right)\right)} \sum_{P \in O\left(P_{n}\right)} \delta_{P}$ converge weakly to $\mu_{v}$. 
Two concepts related to equidistribution are the notions of a sequence of points being generic and small. A sequence $\left\{P_{n}\right\}$ of points in $X(\bar{F})$ is generic if for any subvariety $V \subset X$ there exist $N$ such that $P_{n} \notin V$ for $n>N$. Let $(X, \mathcal{L}, \varphi, \alpha)$ be a polarized dynamical system defined over the number field $F$. A sequence of points $\left\{P_{n}\right\} \in X(\bar{F})$ is said to be small if $h_{\varphi}\left(P_{n}\right)$ converges to zero. The following is an equidistribution theorem in the context of algebraic dynamics.

Theorem 5.2. Let $(X, \mathcal{L}, \varphi, \alpha)$ be a polarized dynamical system defined over the number field $F$. Let $\left\{P_{n}\right\}$ a sequence of points on $X(\bar{F})$ which is generic and small, then for any place $v$ of $F$ the Galois orbits of the sequence $\left\{P_{n}\right\}$ are equidistributed in the analytic space $X_{v}^{a n}$ with respect to the invariant measure $\mu_{\varphi, v}$.

The proof is part of Theorem 5.1 in [29]. Archimedean and non-archimedean places are treated separately. Theorem 4.7 is used to simplify the work over non-Archimedean places. The first $p$-adic equidistribution theorems in the Berkovich setting can be found in [10]. The case of point-wise positive curvature for $\overline{\mathcal{L}}$ was studied in [26] using estimations for the space of global sections. In the arithmetic context the set of global sections is just the unit ball of effective sections in the space of all sections. Let $\overline{\mathcal{L}}$ be a metrized line bundle on $X$. For any section $l \in \Gamma(X, \mathcal{L})_{\mathbb{R}}$, one has a supremum norm $\|l\|_{\text {sup }}=\sup _{z \in X_{\mathbb{C}}}|l(x)|$. Define the invariant $h^{0}(\overline{\mathcal{L}})=\log \#\{l \in \Gamma(X, \mathcal{L}):$ $\|l\|<1\}$. Selecting a Haar measure on $\Gamma(X, \mathcal{L})_{\mathbb{R}}$, we can define the arithmetic volume

$$
\chi(X, \overline{\mathcal{L}})=\log \frac{\operatorname{vol}\left(B_{\text {sup }}\right)}{\operatorname{vol}\left(\Gamma(X, \mathcal{L})_{\mathbb{R}} / \Gamma(X, \mathcal{L})\right)} .
$$

The Theorem of Hilbert and Samuel relates the arithmetic volume with the arithmetic self-intersection for powers of an ample semi-positive metrized line bundle. A line bundle is arithmetically ample if we have the following conditions: $\mathcal{L}_{\sigma}$ is ample in the classical sense for every place $\sigma$ of $F$, the curvature of $\overline{\mathcal{L}}$ is semi-positive, the intersection $\hat{c_{1}}(\overline{\mathcal{L}} \mid Y)^{\operatorname{dim}(Y)}>0$ for all horizontal closed subvariety and $\hat{c_{1}}(\mathcal{L} \mid C) \geq 0$ for any curve on any special fibre.

Theorem 5.3. (Hilbert-Samuel theorem) Let $\overline{\mathcal{L}}$ be an arithmetically ample line bundle on $X$ with a semi-positive adelic metric, and $\operatorname{dim} X=n$, then

$$
\chi\left(X, \overline{\mathcal{L}}^{k}\right) \sim \frac{1}{(n+1) !}\left(\hat{c_{1}}(\overline{\mathcal{L}})^{n+1} \mid X\right) k^{n+1} .
$$

A line bundle $\overline{\mathcal{L}}$ has a semi-positive adelic [31] metric when the metric is limit of semi-positive [30] algebraic metrics with non-negative curvature at archimedean places. The Hilbert-Samuel theorem combined with Minkowski theorem is the key to find global effective sections. 
Lemma 5.4. (Fundamental Inequality) Assume that $\overline{\mathcal{L}}$ is arithmetically ample, with a semi-positive metric. Let $\left\{P_{n}\right\}$ be a generic sequence of points in $X(\bar{F})$. Then,

$$
\liminf h_{\overline{\mathcal{L}}}\left(P_{n}\right) \geq \frac{\hat{\operatorname{deg}}_{X}\left(\hat{c}_{1}(\overline{\mathcal{L}})^{n+1}\right)}{(n+1) \operatorname{deg}_{X}\left(c_{1}(\mathcal{L})^{n}\right)} .
$$

The fundamental inequality follows from a combination of Hilbert-Samuel and th. 4.2 in [30]. It can be applied to the case of a polarized dynamical system $(X, \mathcal{L}, \varphi, \alpha)$, because the $\mathcal{L}$ is equipped with the canonical semipositive metric $\|\cdot\|_{\varphi, v}$. Now, suppose that we denote by $\overline{\mathcal{L}}(\varepsilon f)$ the metrized line bundle obtained from $\overline{\mathcal{L}}$ by multiplying the metric by $\exp (-\varepsilon f)$ at each place. When $X$ is an Abelian variety the metric on the line bundle $\overline{\mathcal{L}}(\varepsilon f)$ is semi-positive for small $\varepsilon$ and we can use the lemma to implement a variational argument. This was the technique used in [26] to prove equidistribution on Abelian varieties. In general, the following theorem of Yuan in [29] will play the role of the Hilbert-Samuel theorem to find effective sections.

Theorem 5.5. Suppose that $\overline{\mathcal{L}}$ and $\bar{M}$ are arithmetically ample. Then, $\chi\left(X,\left(\overline{\mathcal{L}} \otimes \bar{M}^{-1}\right)^{k}\right) \geq \frac{1}{(n+1) !}\left(\hat{c}_{1}(\overline{\mathcal{L}})^{n+1}-(n+1) \hat{c}_{1}(\overline{\mathcal{L}})^{n} \hat{c}_{1}(\bar{M})\right) k^{n+1}+o\left(k^{n+1}\right)$.

The equidistribution theorem in [26] played a crucial role to prove the Bogomolov conjecture for Abelian varieties [32]. A different technique gave equidistribution theorem [6] and Bogomolov conjecture [30] over the multiplicative group $\mathbb{G}_{m}^{n}$. The general case of the Bogomolov conjecture over Algebraic varieties without a group structure is still open.

Conjecture 5.6. (Dynamical Bogomolov Conjecture). Suppose that $(X, \mathcal{L}, \varphi, \alpha)$ is a polarized dynamical system. Let $Y$ be a irreducible closed subvariety of $X$ which is not preperiodic. Then there exist a positive num-

ber $\epsilon>0$, such that the set $\left\{x \in Y(\bar{F}): \hat{h}_{\mathcal{L}}(x)<\epsilon\right\}$ is not Zariski dense in $Y$.

\section{References}

[1] M. Baker, R. Rumely, Potencial theory on the Berkovich projective line, in preparation, 270 pages, (2006).

[2] M. Baker, An introduction to Berkovich analytic spaces and non-archimedean potencial theory on curves (draft 3/16/07), Notes in the Arizona Winter School 2007.

Available at http://swc.math.arizona.edu/aws/07/BakerNotesMarch21.pdf.

[3] M. Baker, R. Rumely, Equidistribution of small points, rational dynamics, and potential theory, Ann. Inst. Fourier (Grenoble) 56 (2006), 625-688.

[4] A. Beauville, Variétés Käehleriennes dont la première classe de Chern est nulle, J. Differential Geom., 87 (1983), 755-782. 
[5] V. G. Berkovich, Spectral theory and analytic geometry over non-Archimedean fields, Mathematical Surveys and Monographs, vol. 33, American Mathematical Society, Providence, R.I., 1990.

[6] Y. Bilu, Limit distribution of small points on algebraic tori, Duke Math. 89 (1997), 465-476.

[7] J-Y. Briend, J. Duval, Deux caractérisations de la mesure d'équilibre d'un endomorphisme de $P^{k}(C)$ IHES, Publ. 93 (2001), 145-159.

[8] H. Brolin, Invariant sets under iteration of rational functions, Ark. Mat. 6 (1965), 103-144.

[9] G. S. Call and J. Silverman, Canonical heights on varieties with morphism, Compositio Math. 89 (1993), 163-205.

[10] A. Chambert-Loir, Mesures et équidistribution sur les espaces de Berkovich., J. für die reine und angewandte Mathematik, vol. 595, p. 215-235.

[11] A. Chambert-Loir and A. Thuillier Formule de Mahler et équidistribution logarithmique., 2006. ArXiv: math.NT/0612556.

[12] J.-P. Demailly, Courants positifs et théorie de l'intersection, Gaz. Math. 53 (1992), 131-158.

[13] J.-P. Demailly, Monge-Ampére operators, Lelong numbers and intersection theory, Complex Analysis and Geometry. Univ. Series in Math., edited by V. Ancona and A. Silva, Plenum Press, New-York (1993).

[14] J.-P. Demailly, Comlex Analytic and Algebraic Geometry, volume I, a paraitre in Grundlehren für Math. Wissenschaften, Springer-Verlag (1997).

[15] V. Guedj, Ergodic properties of rational mappings with large topological degree, Ann. of Math. 161 (2005), 1589-1607.

[16] W. Gubler, Local heights of subvarieties over non-archimedean fields J. Reine. Angew. Math., 498, (1998) 61-113.

[17] W. Gubler, Tropical varieties for non-archimedean analytic spaces, Inventiones mathematicae 169 (2007), 321-376.

[18] R. Hartshorne, Algebraic Geometry, Graduate Texts in Mathematics 52 (1977).

[19] M. Hindry and J. Silverman, Diophantine Geometry: an introduction, Graduate Texts in Mathematics 201 (2000).

[20] S. Kawagushi, Canonical heights, Invariant currents and Dynamical Systems of morphisms associated with Line Bundles., 2000. Archiv: math.NT/0405006.

[21] M. Lyubich, Entropy properties of rational endomorphisms of the Riemann sphere, Ergodic Theory Dyman. Systems 3 (1983), 351-385.

[22] N. Nakayama, Ruled surfaces with nontrivial surjective endomorphisms, Kyushu Journal of Mathematics, 2 (2002).

[23] J. Pineiro, Mahler formula for self maps on $\mathbb{P}^{n}$, 2006. ArXiv: math.NT/0610737.

[24] J. Pineiro, L. Szpiro and T. Tucker, Mahler measure for dynamical systems on $P^{1}$ and intersection theory on a syngular arithmetic surface in Geometric Methods in Algebra and Number Theory (edited by F. Bogomolov and Y. Tschinkel), Progress in Mathematics 235, Birkhäuser, 2004, pp. 219-250.

[25] J. Silverman, Rational points on K3 surfaces: a new canonical height, Invent. Math. 105 (1991), 347-373.

[26] L. Szpiro, E. Ullmo, and S. Zhang, Equirépartition des petits points, Invent. Math. 127 (1997), 337-347.

[27] L. Szpiro, Cours de géométrie arithmétique, Orsay preprint. Available at http://math.gc.cuny.edu/faculty/szpiro/papers.

[28] A. Thuillier, Théorie du potentiel sur les courbes en gééometrie analytique non archimédienne. Applications à la théorie d'Arakelov. Ph.D. thesis, Université ded Rennes, 2006. 
[29] X. Yuan, Big line bundles on arithmetic varieties, 2006. arXiv: math.NT/0612424.

[30] S. Zhang, Positive line bundles on arithmetic varieties, J. Amer. Math. Soc. 8 (1995), 187-221.

[31] S. Zhang, Small points and adelic metrics, J. Algebraic Geometry 4 (1995), 281300.

[32] S. Zhang, Equidistribution of small points on abelian varieties, Ann. of Math. (2) 147 (1998), no. 1, 159-165.

[33] S. Zhang, Distributions in Algebraic Dynamics, Survey in Differential Geometry, vol 10, International Press, 2006, pp. 381-430. 